VOL. 60 (1999) [353-364]

\title{
NONLINEAR HEMIVARIATIONAL INEQUALITIES AT RESONANCE
}

\author{
Leszek Gasiński AND Nikolaos S. Papageorgiou
}

In this paper we consider nonlinear hemivariational inequalities involving the $p$ Laplacian at resonance. We prove the existence of a nontrivial solution. Our approach is variational based on the critical point theory for nonsmooth, locally Lipschitz functionals due to Chang.

\section{INTRODUCTION}

Let $Z \subset \mathbb{R}^{N}$ be a bounded domain with a $\mathcal{C}^{1}$-boundary $\Gamma$. In this paper we study the following nonlinear hemivariational inequality at resonance:

$$
\left\{\begin{array}{l}
-\operatorname{div}\left(\|\nabla x(z)\|^{p-2} \nabla x(z)\right)-\lambda_{1}|x(z)|^{p-2} x(z) \in \partial j(z, x(z)) \\
\text { almost everywhere on } Z \\
\left.x\right|_{\Gamma}=0,
\end{array}\right.
$$

where $2 \leqslant p<+\infty$. Here $\lambda_{1}$ is the first eigenvalue of the $p$-Laplacian $-\Delta_{p} x \stackrel{d f}{=}$ $-\operatorname{div}\left(\|\nabla x\|^{p-2} \nabla x\right)$ with Dirichlet boundary condition (that is, of the operator $\left.\left(-\Delta_{p}, W_{0}^{1, p}(Z)\right)\right), j: Z \times \mathbb{R} \longmapsto \mathbb{R}$ is a functional measurable in $z \in Z$ which is locally Lipschitz in $\zeta \in \mathbb{R}$ and $\partial j(z, \zeta)$ denotes the subdifferential of $j(z, \cdot)$ in the sense of Clarke [7] (generalised subdifferential). Our starting point is the recent paper by Goeleven-Motreanu-Panagiotopoulos [9], where the authors considered eigenvalue problems for semilinear hemivariational inequalities. In that paper the authors proved the existence of multiple solutions for the eigenvalue problems they examined, and also mentioned as an open problem for future research the resonant case. Our work here can also be viewed as the continuation of the recent work by the authors (see Gasiński-Papageorgiou [8]) where the work of Goeleven-Motreanu-Panagiotopoulos [9] was extended to eigenvalue problems for nonlinear hemivariational inequalities involving the $p$-Laplacian. In this paper we assume that the generalised subdifferential of $j(z, \zeta)$ has nonzero limits as $\zeta \rightarrow \pm \infty$. Assuming that $j(\cdot, 0) \in L^{\infty}(Z)$, by the Lebourg mean value theorem (see

Received 25th January, 1999

This paper was written when the second author visited the Jagiellonian University. He wants to thank Professor Z. Denkowski and Professor S. Migórski of the Department of Computer Science for arranging this visit and for their warm hospitality.

Copyright Clearance Centre, Inc. Serial-fee code: 0004-9727/99 \$A2.00+0.00. 
Clarke [7, Theorem 2.3.7, p.41]) we infer that $j(z, \zeta)$ (the generalised potential) goes to infinity as $\zeta \rightarrow \pm \infty$. This is the case studied by Ahmad-Lazer-Paul [1] and Rabinowitz [14], where $p=2$ (semilinear case) and $j(z, \cdot) \in \mathcal{C}^{1}$. The case of finite limits for $j(z, \zeta)$ as $\zeta \rightarrow \pm \infty$ was examined by Landesman-Lazer [10] (the pioneering work in this area), Thews [17], Ward [18] and Benci-Bartolo-Fortunato [3], where $p=2$ and $j(z, \cdot) \in \mathcal{C}^{1}$. We should mention that Benci-Bartolo-Fortunato called their problem "strongly resonant".

Hemivariational inequalities arise in physical problems when we deal with nonconvex nonsmooth energy functionals. Such functions appear quite often in mechanics and engineering if one wants to consider more realistic mechanical laws of a nonmonotone, multivalued nature. For concrete applications of hemivariational inequalities to problems in mechanics we refer to the book of Panagiotopoulos [13], Naniewicz-Panagiotopoulos [12] and references therein.

Our approach is variational based on the critical point theory for nonsmooth Lipschitz functionals as this was developed by Chang [6]. For the convenience of the reader in the next section we recall the basic notions and facts from that theory, which we shall need in the sequel.

\section{Preliminaries}

Let $X$ be a Banach space and $X^{*}$ its topological dual. A function $f: X \mapsto \mathbb{R}$ is said to be locally Lipschitz, if for every $x \in X$ there exists a neighbourhood $U$ of $x$ and a constant $k>0$ depending on $U$ such that $|f(z)-f(y)| \leqslant k\|z-y\|$ for all $z, y \in U$. It is well known from convex analysis that a proper, convex and lower semicontinuous function $g: X \longmapsto \mathbb{\mathbb { R }}=\mathbb{R} \cup\{+\infty\}$ is locally Lipschitz in the interior of its domain dom $g=\{x \in X: g(x)<+\infty\}$. In analogy with the directional derivative of a convex function, we define the generalised directional derivative of a locally Lipschitz function $f$ at $x \in X$ in the direction $h \in X$, by

$$
f^{0}(x ; h)=\limsup _{\substack{x^{\prime} \rightarrow 0 \\ \lambda \succ 0}} \frac{f\left(x+x^{\prime}+\lambda h\right)-f\left(x+x^{\prime}\right)}{\lambda} .
$$

It is easy to check that $X \ni h \longmapsto f^{0}(x ; h) \in \mathbb{R}$ is sublinear and continuous and $\left|f^{0}(x ; h)\right| \leqslant k\|h\|$. So by the Hahn-Banach theorem $f^{0}(x ; \cdot)$ is the support function of the nonempty, convex and $w^{*}$-compact set

$$
\partial f(x)=\left\{x^{*} \in X^{*}:\left(x^{*}, h\right) \leqslant f^{0}(x, h) \text { for all } h \in X\right\}
$$

known as the "generalised subdifferential" of $f$ at $x$. Note that for every $x^{*} \in \partial f(x)$ we have $\left\|x^{*}\right\| \leqslant k$. Also if $f, g: X \longmapsto \mathbb{R}$ are locally Lipschitz functions, then $\partial(f+g) \subseteq$ $\partial f(x)+\partial g(x)$ and $\partial(\lambda f)(x)=\lambda \partial f(x)$ for all $\lambda \in \mathbb{R}$. Moreover, if $f: X \longmapsto \mathbb{R}$ is convex then it is well-known that $f$ is locally Lipschitz and the subdifferential of $f$ in the 
sense of convex analysis coincides with the generalised subdifferential introduced above. Moreover, if $f$ is strictly differentiable at $x$ (in particular if $f$ is continuously Gateaux differentiable at $x$ ), then $\partial f(x)=\left\{f^{\prime}(x)\right\}$.

Let $f: X \longmapsto \mathbb{R}$ be a locally Lipschitz function on a Banach space $X$. A point $x \in X$ is said to be a "critical point" of $f$, if $0 \in \partial f(x)$. It is easy to see that, if $x \in X$ is a local minimum of $f$, then $x$ is a critical point. We say that $f$ satisfies the "Palais-Smale condition" ((PS)-condition) if any sequence $\left\{x_{n}\right\}_{n \geqslant 1} \subseteq X$ along which $\left\{f\left(x_{n}\right)\right\}_{n \geqslant 1}$ is bounded and $m\left(x_{n}\right) \stackrel{d f}{=} \min \left\{\left\|x^{*}\right\|: x^{*} \in \partial f\left(x_{n}\right)\right\} \longrightarrow 0$ as $n \rightarrow+\infty$, has a strongly convergent subsequence. If $f \in \mathcal{C}^{1}(X)$, then since $\partial f\left(x_{n}\right)=\left\{f^{\prime}\left(x_{n}\right)\right\}$, we see that the above definition of the Palais-Smale condition coincides with the classical one (see Rabinowitz [15]).

The first theorem is due to Chang [6] and extends to a nonsmooth setting the well known "mountain pass theorem" due to Ambrosetti-Rabinowitz [2].

Theorem 1. If $X$ is a reflexive Banach space, $R: X \longmapsto \mathbb{R}$ is locally Lipschitz functional which satisfies the (PS)-condition and for some $\rho>0$ and $y \in X$ with $\|y\|>\rho$ we have

$$
\max \{R(0), R(y)\}<\inf _{\|x\|=\rho}\{R(x)\},
$$

then $R$ has a nontrivial critical point $x \in X$ such that the critical value $c=R(x)$ is characterised by the following minimax principle

$$
c=\inf _{\gamma \in \Gamma} \max _{0 \leqslant \tau \leqslant 1}\{R(\gamma(\tau))\}
$$

where $\Gamma=\{\gamma \in \mathcal{C}([0,1], X): \gamma(0)=0, \gamma(1)=y\}$.

In $(H V I)$ appears the first eigenvalue $\lambda_{1}$ of $\left(-\Delta_{p}, W_{0}^{1, p}(Z)\right)$. This is the least real number $\lambda$ for which the problem

$(E P) \quad\left\{\begin{array}{l}-\operatorname{div}\left(\|\nabla x(z)\|^{p-2} \nabla x(z)\right)=\lambda|x(z)|^{p-2} x(z) \text { almost everywhere on } Z \\ \left.x\right|_{\Gamma}=0\end{array}\right.$

has a nontrivial solution. This first eigenvalue $\lambda_{1}$ is positive, isolated and simple (that is, the associated eigenfunctions are constant multiples of each other). Futhermore we have a variational characterisation of $\lambda_{1}$ via the Rayleigh quotient, that is,

$$
\lambda_{1}=\min \left\{\frac{\|\nabla x\|_{p}^{p}}{\|x\|_{p}^{p}}: x \in W_{0}^{1, p}(Z), x \neq 0\right\} .
$$

This minimum is realised at the normalised eigenfunction $u_{1}$. Note that if $u_{1}$ minimises the Rayleigh quotient, then so does $\left|u_{1}\right|$ and so we infer that the first eigenfunction $u_{1}$ does not change sign on $Z$. In fact we can show that $u_{1}(z) \neq 0$ almost everywhere on $Z$ and so we can assume that $u_{1}>0$ almost everywhere on $Z$. For details we refer to Lindqvist [11]. 


\section{Existence of Solutions}

We start by introducing our hypotheses on the function $j(z, \zeta)$.

$H(j) j: Z \times \mathbb{R} \longmapsto \mathbb{R}$ is a functional such that:

(i) for all $\zeta \in \mathbb{R}: Z \ni z \longmapsto j(z, \zeta) \in \mathbb{R}$ is measurable;

(ii) for almost all $z \in Z: \mathbb{R} \ni \zeta \longmapsto j(z, \zeta) \in \mathbb{R}$ is locally Lipschitz;

(iii) for almost all $z \in Z$, all $\zeta \in \mathbb{R}$ and all $v \in \partial j(z, \zeta)$ we have $|v| \leqslant a(z)$ with some $a \in L^{\infty}(Z)$;

(iv) $j(\cdot, 0) \in L^{\infty}(Z)$ and $\int_{Z} j(z, 0) d z \geqslant 0$;

(v) for almost all $z \in Z$ and all $v(z, \zeta) \in \partial j(z, \zeta): v(\cdot, \zeta)$ is measurable, we have $v(z, \zeta) \longrightarrow v_{-}(z)$ as $\zeta \rightarrow-\infty$ and $v(z, \zeta) \longrightarrow v_{+}(z)$ as $\zeta \rightarrow+\infty$, where $v_{-}$ and $v_{+}$are measurable and $v_{-}(z) \leqslant 0 \leqslant v_{+}(z)$ almost everywhere on $Z$ with strict inequalities on a set of positive Lebesgue measurable;

(vi) there exists $\mu>\lambda_{1}$ such that

$$
\limsup _{\zeta \rightarrow 0} \frac{p j(z, \zeta)}{|\zeta|^{p}}<-\mu
$$

uniformily for almosta all $z \in Z$.

We introduce two functionals $K: W_{0}^{1, p}(Z) \longmapsto \mathbb{R}_{+}$and $L: W_{0}^{1, p}(Z) \longmapsto \mathbb{R}$ defined by

$$
\begin{aligned}
& K(x) \stackrel{d f}{=} \frac{1}{p}\|\nabla x\|_{p}^{p} \\
& L(x) \stackrel{d f}{=} \frac{\lambda_{1}}{p}\|x\|_{p}^{p}+\int_{Z} j(z, x(z)) d z .
\end{aligned}
$$

Clearly $K \in \mathcal{C}^{1}\left(W_{0}^{1, p}(Z)\right)$ and is convex (thus $K$ is locally Lipschitz). Using [7, Theorem 2.7 .5$, p.83], we see that $L$ is locally Lipschitz too. Set $R=K-L$. Then $R: W_{0}^{1, p}(Z) \longmapsto$ $\mathbb{R}$ is locally Lipschitz.

LEMMA 2. If hypotheses $H(j)$ hold, then $R$ satisfies the $(P S)$-condition.

PROOF: Let $\left\{x_{n}\right\}_{n \geqslant 1} \subseteq W_{0}^{1, p}(Z)$ be a sequence such that $\left\{R\left(x_{n}\right)\right\}_{n \geqslant 1}$ is bounded and $m\left(x_{n}\right) \longrightarrow 0$ as $n \rightarrow+\infty$. Then there exists $M_{1}>0$ such that for all $n \geqslant 1$ we have $\left|R\left(x_{n}\right)\right| \leqslant M_{1}$, so

$$
-M_{1} \leqslant \frac{1}{p}\left\|\nabla x_{n}\right\|_{p}^{p}-\frac{\lambda_{1}}{p}\left\|x_{n}\right\|_{p}^{p}-\int_{Z} j\left(z, x_{n}(z)\right) d z \leqslant M_{1}
$$

Suppose that the sequence $\left\{x_{n}\right\}_{n \geqslant 1} \subset W_{0}^{1, p}(Z)$ was unbounded. Then by passing to a subsequence if necessary, we may assume that $\left\|x_{n}\right\| \longrightarrow+\infty$ as $n \rightarrow+\infty$. Set $y_{n}=$ 
$\left(x_{n} /\left\|x_{n}\right\|\right)$ for $n \geqslant 1$. By passing to a further subsequence, if necessary, we may assume that

$$
\begin{aligned}
y_{n} & \longrightarrow y \text { weakly in } W_{0}^{1, p}(Z), \\
y_{n} & \longrightarrow y \text { in } L^{p}(Z), \\
y_{n}(z) & \longrightarrow y(z) \text { almost everywhere on } Z \text { as } n \rightarrow+\infty,
\end{aligned}
$$

and $\left|y_{n}(z)\right| \leqslant k(z)$ almost everywhere on $Z$ with $k \in L^{p}(Z)$ (see [4, Theorem IV.9, p.58]).

Dividing (1) by $\left\|x_{n}\right\|^{p}$, we obtain

$$
-\frac{M_{1}}{\left\|x_{n}\right\|^{p}} \leqslant \frac{1}{p}\left\|\nabla y_{n}\right\|_{p}^{p}-\frac{\lambda_{1}}{p}\left\|y_{n}\right\|_{p}^{p}-\int_{Z} \frac{j\left(z, x_{n}(z)\right)}{\left\|x_{n}\right\|^{p}} d z \leqslant \frac{M_{1}}{\left\|x_{n}\right\|^{p}} .
$$

Let us establish the asymptotic behaviour of the integral $\int_{Z}\left(j\left(z, x_{n}(z)\right) /\left\|x_{n}\right\|^{p}\right) d z$. By the Lebourg mean value theorem (see Clarke [7, Theorem 2.3.7, p.47], for almost all $z \in Z$, all $\zeta \in \mathbb{R}$ and for some $v \in \partial j(z, \bar{\zeta}), \bar{\zeta}=\alpha \zeta, 0<\alpha<1$, we have, using hypothesis $H(j)($ iii), that

$$
|j(z, \zeta)-j(z, 0)|=|\langle v, \bar{\zeta}\rangle| \leqslant a(z)|\zeta|
$$

and using $H(j)$ (iv) we obtain

$$
|j(z, \zeta)| \leqslant|j(z, 0)|+a(z)|\zeta| \leqslant a_{1}+a_{2}|\zeta|
$$

for some $a_{1}, a_{2}>0$. So we can write that

$$
\begin{aligned}
\left|\int_{Z} \frac{j\left(z, x_{n}(z)\right)}{\left\|x_{n}\right\|^{p}} d z\right| & \leqslant \int_{Z} \frac{\left|j\left(z, x_{n}(z)\right)\right|}{\left\|x_{n}\right\|^{p}} d z \leqslant \int_{Z} \frac{a_{1}+a_{2}\left|x_{n}(z)\right|}{\left\|x_{n}\right\|^{p}} d z \\
& \leqslant \frac{a_{3}}{\left\|x_{n}\right\|^{p}}+\frac{a_{4}}{\left\|x_{n}\right\|^{p-1}}
\end{aligned}
$$

with $a_{3}=a_{1}|Z|$ and $a_{4}=a_{2} \int_{Z}|k(z)| d z$. So

$$
\int_{Z} \frac{j\left(z, x_{n}(z)\right)}{\left\|x_{n}\right\|^{p}} d z \rightarrow 0 \text { as } n \rightarrow+\infty .
$$

Thus if we pass to the limit as $n \rightarrow+\infty$ in (2), we obtain

$$
\frac{1}{p} \liminf _{n \rightarrow+\infty}\left\|\nabla y_{n}\right\|_{p}^{p}=\frac{\lambda_{1}}{p}\|y\|_{p}^{p}
$$

But since $\nabla y_{n} \rightarrow \nabla y$ weakly in $L^{p}\left(Z, \mathbb{R}^{N}\right)$ as $n \rightarrow+\infty$ (recall that $y_{n} \longrightarrow y$ weakly in $W_{0}^{1, p}(Z)$ ), from the weak lower semicontinuity of the norm functional, we have that

$$
\frac{1}{p}\|\nabla y\|_{p}^{p} \leqslant \liminf _{n \rightarrow+\infty} \frac{1}{p}\left\|\nabla y_{n}\right\|_{p}^{p}
$$


and so

$$
\frac{1}{p}\|\nabla y\|_{p}^{p} \leqslant \frac{\lambda_{1}}{p}\|y\|_{p}^{p}
$$

On the other hand from the variational characterisation of $\lambda_{1}$ (Rayleigh quotient, see section 2), we have

$$
\frac{\lambda_{1}}{p}\|y\|_{p}^{p} \leqslant \frac{1}{p}\|\nabla y\|_{p}^{p}
$$

So finally we deduce that

$$
\|\nabla y\|_{p}^{p}=\lambda_{1}\|y\|_{p}^{p} .
$$

Also, by passing to the limit in (2), we obtain

$$
\limsup _{n \rightarrow+\infty} \frac{1}{p}\left\|\nabla y_{n}\right\|_{p}^{p} \leqslant \frac{\lambda_{1}}{p}\|y\|_{p}^{p}=\frac{1}{p}\|\nabla y\|_{p}^{p} \leqslant \liminf _{n \rightarrow+\infty} \frac{1}{p}\left\|\nabla y_{n}\right\|_{p}^{p},
$$

so

$$
\left\|\nabla y_{n}\right\|_{p} \longrightarrow\|\nabla y\|_{p} \quad \text { as } n \rightarrow+\infty .
$$

Since $\nabla y_{n} \longrightarrow \nabla y$ weakly in $L^{p}\left(Z, \mathbb{R}^{N}\right)$ as $n \rightarrow+\infty$ and $L^{p}\left(Z, \mathbb{R}^{N}\right)$ is uniformly convex, we infer that $\nabla y_{n} \longrightarrow \nabla y$ in $L^{p}\left(Z, \mathbb{R}^{N}\right)$ as $n \rightarrow+\infty$. Hence $y_{n} \longrightarrow y$ in $W_{0}^{1, p}(Z)$ as $n \rightarrow+\infty$. But $\left\|y_{n}\right\|=1$ for $n \geqslant 1$, so $\|y\|=1$, thus $y \neq 0$. Therefore from the equality $\|\nabla y\|_{p}^{p}=\lambda_{1}\|y\|_{p}^{p}$, it follows that $y= \pm u_{1}$.

Without loss of generality we may assume that $y=+u_{1}$ (the analysis is similar if $\left.y=-u_{1}\right)$. So from section 2 we know that $y(z)=u_{1}(z)>0$ almost everywhere on $\mathrm{Z}$. Now let $x_{n}^{*} \in \partial R\left(x_{n}\right)$ for $n \geqslant 1$, be such that $m\left(x_{n}\right)=\left\|x_{n}^{*}\right\|$. The existence of such an element follows from the fact that $\partial R\left(x_{n}\right)$ is a nonempty weakly compact subset of $W^{-1, q}(Z)=W_{0}^{1, p}(Z)^{*}$ (see section 2) and the weak lower semicontinuity of the norm functional. Consider the operator $A: W_{0}^{1, p}(Z) \longmapsto W^{-1, q}(Z)$ defined by

$$
\langle A x, u\rangle=\int_{Z}\|\nabla x(z)\|^{p-2}(\nabla x(z), \nabla u(z))_{\mathbb{R}^{n}} d z \quad \forall x, u \in W_{0}^{1, p}(Z) .
$$

Here by $\langle\cdot, \cdot\rangle$ we denote the duality brackets for the pair $\left(W_{0}^{1, p}(Z), W^{-1, q}(Z)\right)$. It is easy to check that $A$ is monotone, hemicontinuous, hence maximal monotone. So it is also generalised pseudomonotone (see Browder-Hess [5]). Then we have

$$
x_{n}^{*}=A x_{n}-\lambda_{1}\left\|x_{n}\right\|^{p-2} x_{n}-v_{n},
$$

where $v_{n} \in \partial \psi\left(x_{n}\right), n \geqslant 1$, with $\psi: W_{0}^{1, p}(Z) \rightarrow \mathbb{R}$ being defined by $\psi(x)=$ $\int_{Z} j(z, x(z)) d z$. We know that, if $v \in \partial \psi(x)$, then $v(z) \in \partial j(z, x(z))$ almost everywhere on $Z$ (see Clarke [7]). Note that $\psi=\left.\widehat{\psi}\right|_{W_{0}^{1, p}(Z)}$ where $\hat{\psi}: L^{p}(Z) \longrightarrow \mathbb{R}$ is defined by $\widehat{\psi}(x)=\int_{Z} j(z, x(z)) d z$. So invoking [6, Theorem 2.2], we have thet $\partial \psi(x) \subseteq \partial \widehat{\psi}(x) \subseteq L^{q}(Z)$. Hence $v_{n} \in L^{q}(Z)$ for all $n \geqslant 1$. 
From the choice of the sequence $\left\{x_{n}\right\}_{n \geqslant 1}$ we have that $\left|R\left(x_{n}\right)\right| \leqslant M_{1}$ and

$$
\left|\left\langle x_{n}^{*}, u\right\rangle\right| \leqslant \varepsilon_{n}\|u\| \quad \forall u \in W_{0}^{1, p}(Z) \text { with } \varepsilon_{n} \searrow 0
$$

From (1), we have

$$
-p M_{1} \leqslant\left\|\nabla x_{n}\right\|_{p}^{p}-\lambda_{1}\left\|x_{n}\right\|_{p}^{p}-p \int_{Z} j\left(z, x_{n}(z)\right) d z \leqslant p M_{1}
$$

and taking $u=x_{n}$ in (3), we have

$$
-\varepsilon_{n}\left\|x_{n}\right\| \leqslant-\left\langle A x_{n}, x_{n}\right\rangle+\lambda_{1}\left\|x_{n}\right\|_{p}^{p}+\int_{Z} v_{n}(z) x_{n}(z) d z \leqslant \varepsilon_{n}\left\|x_{n}\right\| .
$$

We add (4) and (5). Because $\left\langle A x_{n}, x_{n}\right\rangle=\left\|\nabla x_{n}\right\|_{p}^{p}$, we have

$$
-p M_{1}-\varepsilon_{n}\left\|x_{n}\right\| \leqslant \int_{Z}\left(v_{n}(z) x_{n}(z)-p j\left(z, x_{n}(z)\right)\right) d z \leqslant p M_{1}+\varepsilon_{n}\left\|x_{n}\right\| .
$$

Dividing by $\left\|x_{n}\right\|$ we obtain

$$
-\frac{p M_{1}}{\left\|x_{n}\right\|}-\varepsilon_{n} \leqslant \int_{Z}\left(v_{n}(z) y_{n}(z)-\frac{p j\left(z, x_{n}(z)\right)}{\left\|x_{n}\right\|}\right) d z \leqslant \frac{p M_{1}}{\left\|x_{n}\right\|}+\varepsilon_{n} .
$$

We know that $y_{n}(z) \longrightarrow u_{1}(z)>0$ almost everywhere on $Z$ as $n \rightarrow+\infty$ and so $x_{n}(z) \longrightarrow+\infty$ almost everywhere on $Z$ as $n \rightarrow+\infty$. Then because of hypothesis $H(j)(v)$ and since $v_{n}(z) \in \partial j\left(z, x_{n}(z)\right)$ almost everywhere on $Z$, we have that $v_{n}(z) \longrightarrow v_{+}(z)$ almost everywhere on $Z$ as $n \rightarrow+\infty$. Hence by the Lebesgue dominated convergence theorem, we have that $\int_{Z} v_{n}(z) y_{n}(z) d z \rightarrow \int_{Z} v_{+}(z) u_{1}(z) d z$ as $n \rightarrow+\infty$. Next let $N \subseteq Z$ be the Lebesgue-null set outside of which we have $x_{n}(z) \rightarrow+\infty$ as $n \rightarrow+\infty$ and hypothesis $H(j)(v)$ holds. Fix $z \in Z \backslash N$. For a given $0<\varepsilon<1$, from the Lebourg mean value theorem, we have

$$
j\left(z, x_{n}(z)\right)=j\left(z, \varepsilon x_{n}(z)\right)+w_{n}(z)(1-\varepsilon) x_{n}(z),
$$

with $w_{n}(z) \in \partial j\left(z, u_{n}(z)\right)$, where $u_{n}(z)=\left(1-\alpha_{n}\right) x_{n}(z)+\alpha_{n} \varepsilon x_{n}(z)$, with $0<\alpha_{n}<1$, for $n \geqslant 1$. Hence $u_{n}(z)=x_{n}(z)-\alpha_{n}(1-\varepsilon) x_{n}(z) \geqslant x_{n}(z)-(1-\varepsilon) x_{n}(z)=\varepsilon x_{n}(z)$. Since $x_{n}(z) \longrightarrow+\infty$ as $n \rightarrow+\infty$ and $\varepsilon>0$, we have that $u_{n}(z) \rightarrow+\infty$ as $n \rightarrow+\infty$ and so $w_{n}(z) \longrightarrow v_{+}(z)$ as $n \rightarrow+\infty$ (see hypothesis $H(j)(v)$ ). Now let $n_{0}=n_{0}(\varepsilon, z) \geqslant 1$ be such that for all $n \geqslant n_{0}$ we have $x_{n}(z)>0$ and $\left|w_{n}(z)-v_{+}(z)\right| \leqslant \varepsilon$. We have, for $n \geqslant 1$, that

$$
\frac{p j\left(z, x_{n}(z)\right)}{x_{n}(z)}=\frac{p j\left(z, \varepsilon x_{n}(z)\right)}{x_{n}(z)}+\frac{p w_{n}(z)(1-\varepsilon) x_{n}(z)}{x_{n}(z)} .
$$


Recall that $\left|j\left(z, \varepsilon x_{n}(z)\right)\right| \leqslant a_{1}+a_{2} \varepsilon\left|x_{n}(z)\right|$, while $-\varepsilon+v_{+}(z) \leqslant w_{n}(z) \leqslant \varepsilon+v_{+}(z)$. So for $n \geqslant n_{0}$ we can write that

$$
\begin{aligned}
\frac{-p a_{1}-p a_{2} \varepsilon x_{n}(z)}{x_{n}(z)} & +\frac{p\left(-\varepsilon+v_{+}(z)\right)(1-\varepsilon) x_{n}(z)}{x_{n}(z)} \\
& \leqslant \frac{p j\left(z, x_{n}(z)\right)}{x_{n}(z)} \leqslant \frac{p a_{1}+p a_{2} \varepsilon x_{n}(z)}{x_{n}(z)}+\frac{p\left(\varepsilon+v_{+}(z)\right)(1-\varepsilon) x_{n}(z)}{x_{n}(z)} .
\end{aligned}
$$

Since $\varepsilon>0$ was arbitrary, from the above inequalities which are valid for $n \geqslant n_{0}$, we infer that

$$
\frac{p j\left(z, x_{n}(z)\right)}{x_{n}(z)} \longrightarrow p v_{+}(z) \quad \text { as } n \rightarrow+\infty
$$

Therefore it follows that

$$
\begin{aligned}
\int_{Z} \frac{p j\left(z, x_{n}(z)\right)}{\left\|x_{n}\right\|} d z & =\int_{Z} \frac{p j\left(z, x_{n}(z)\right)}{x_{n}(z)} \cdot \frac{x_{n}(z)}{\left\|x_{n}\right\|} d z \\
& =\int_{Z} \frac{p j\left(z, x_{n}(z)\right)}{x_{n}(z)} y_{n}(z) d z \rightarrow p \int_{Z} v_{+}(z) u_{1}(z) d z \text { as } n \rightarrow+\infty
\end{aligned}
$$

So if we pass to the limit as $n \rightarrow+\infty$ in (6), we obtain

$$
(1-p) \int_{Z} v_{+}(z) u_{1}(z) d z=0
$$

so

$$
\int_{Z} v_{+}(z) u_{1}(z) d z=0
$$

But recall that $u_{1}(z)>0$ almost everywhere on $Z$ and $v_{+}(z) \geqslant 0$ almost everywhere on $Z$ with strict inequality on a set of positive Lebesgue measure (see hypothesis $H(j)(v)$ ). Thus $\int_{Z} v_{+}(z) u_{1}(z) d z>0$, a contradiction. This implies that $\left\{x_{n}\right\}_{n} \geqslant 1 \subset W_{0}^{1, p}(Z)$ is bounded. So by passing to a subsequence if necessary we may assume that $x_{n} \longrightarrow x$ weakly in $W_{0}^{1, p}(Z), x_{n} \longrightarrow x$ in $L^{p}(Z), x_{n}(z) \longrightarrow x(z)$ almost everywhere on $Z$ and $\left|x_{n}(z)\right| \leqslant k_{1}(z)$ almost everywhere on $Z$ with $k_{1} \in L^{p}(Z)$.

Putting $u=x_{n}-x$ in (3) we obtain

$$
\begin{aligned}
-\varepsilon_{n}\left\|x_{n}-x\right\| \leqslant & \left\langle A x_{n}, x_{n}-x\right\rangle-\lambda_{1} \int_{Z}\left|x_{n}(z)\right|^{p-2} x_{n}(z)\left(x_{n}-x\right)(z) d z \\
& -\int_{Z} v_{n}(z)\left(x_{n}-x\right)(z) d z \leqslant \varepsilon_{n}\left\|x_{n}-x\right\| .
\end{aligned}
$$

Note that

$$
\begin{gathered}
\lambda_{1} \int_{Z}\left|x_{n}(z)\right|^{p-2} x_{n}(z)\left(x_{n}-x\right)(z) d z \longrightarrow 0 \quad \text { as } n \rightarrow+\infty, \text { and } \\
\int_{Z} v_{n}(z)\left(x_{n}-x\right)(z) d z \longrightarrow 0 \quad \text { as } n \rightarrow+\infty
\end{gathered}
$$


So from (7), we have

$$
\limsup _{n \rightarrow+\infty}\left\langle A x_{n}, x_{n}-x\right\rangle \leqslant 0 .
$$

But we already know that $A$ is generalised pseudomonotone. So we have

$$
\left\langle A x_{n}, x_{n}\right\rangle \longrightarrow\langle A x, x\rangle \text { as } n \rightarrow+\infty,
$$

so

$$
\left\|\nabla x_{n}\right\|_{p}^{p} \rightarrow\|\nabla x\|_{p}^{p} \text { as } n \rightarrow+\infty .
$$

Also $\nabla x_{n} \longrightarrow \nabla x$ weakly in $L^{p}\left(Z, \mathbb{R}^{N}\right)$ as $n \rightarrow+\infty$. Since $L^{p}\left(Z, \mathbb{R}^{N}\right)$ is uniformly convex, we conclude that $\nabla x_{n} \longrightarrow \nabla x$ in $L^{p}\left(Z, \mathbb{R}^{N}\right)$ as $n \rightarrow+\infty$ and so $x_{n} \longrightarrow x$ in $W_{0}^{1, p}(Z)$ as $n \rightarrow+\infty$. Thus $R$ satisfies the $(P S)$-condition.

LEMMA 3. If hypotheses $H(j)$ hold, then there exist $\beta_{1}, \beta_{2}>0$ such that for all $x \in W_{0}^{1, p}(Z)$, we have

$$
R(x) \geqslant \beta_{1}\|x\|^{p}-\beta_{2}\|x\|^{\vartheta}
$$

with $p<\vartheta \leqslant p^{*}=N p /(N-p)$.

Proof: Let $\varepsilon>0$ be such that $\lambda_{1}+\varepsilon<\mu$. From hypothesis $H(j)$ (vi) we can find $\delta>0$ such that for almost all $z \in Z$ and all $\zeta$ such that $|\zeta| \leqslant \delta$ we have

$$
j(z \zeta) \leqslant \frac{1}{p}(-\mu+\varepsilon)|\zeta|^{p}
$$

On the other hand from the proof of Lemma 2 we know that for almost all $z \in Z$ and all $\zeta$ such that $|\zeta|>\delta$ we have

$$
|j(z, \zeta)| \leqslant a_{1}+a_{2}|\zeta|
$$

with some $a_{1}, a_{2}>0$. Thus for almost all $z \in Z$ and all $\zeta \in \mathbb{R}$ we have

$$
j(z, \zeta) \leqslant \frac{1}{p}(-\mu+\varepsilon)|\zeta|^{p}+\gamma|\zeta|^{\vartheta},
$$

with $\gamma=\left(a_{1}+a_{2} \delta\right) \delta^{-\vartheta}+(\mu-\varepsilon) \delta^{p-\vartheta} / p$ and $p<\vartheta \leqslant p^{*}=N p /(N-p)$. Using this we obtain that

$$
\begin{aligned}
R(x) & =\frac{1}{p}\|\nabla x\|_{p}^{p}-\frac{\lambda_{1}}{p}\|x\|_{p}^{p}-\int_{Z} j(z, x(z)) d z \\
& \geqslant \frac{1}{p}\|\nabla x\|_{p}^{p}-\frac{\lambda}{p}\|x\|_{p}^{p}+\frac{1}{p}(\mu-\varepsilon)\|x\|_{p}^{p}-\gamma\|x\|_{\theta}^{\theta} \\
& =\frac{1}{p}\|\nabla x\|_{p}^{p}-\frac{1}{p}\left(\lambda_{1}-\mu+\varepsilon\right)\|x\|_{p}^{p}-\gamma\|x\|_{\theta}^{\theta} .
\end{aligned}
$$

From the choice of $\varepsilon$, we have $-(1 / p)\left(\lambda_{1}-\mu+\varepsilon\right)\|x\|_{p}^{p}>0$, so

$$
R(x) \geqslant \frac{1}{p}\|\nabla x\|_{p}^{p}-\gamma\|x\|_{\theta}^{\theta} .
$$


Because $\vartheta<p^{*}=N p /(N-p)$, from the Sobolev embedding theorem we have that $W_{0}^{1, p}(Z)$ is embedded continuously in $L^{\theta}(Z)$. So using the Poincare inequality, it follows that

$$
R(x) \geqslant \beta_{1}\|x\|^{p}-\beta_{2}\|x\|^{\vartheta},
$$

for some $\beta_{1}, \beta_{2}>0$ and all $x \in W_{0}^{1, p}(Z)$.

Using Lemmas 2 and 3 we have the following existence theorem for problem $(H V I)$.

THEOREM 4. If hypothesis $H(j)$ hold, then the problem (HVI) has a nontrivial solution.

Proof: From Lemma 3 we know that there exist $\beta_{1}, \beta_{2}>0$ such that for all $x \in$ $W_{0}^{1, p}(Z)$ we have

$$
R(x) \geqslant \beta_{1}\|x\|^{p}-\beta_{2}\|x\|^{\vartheta}=\beta_{1}\|x\|^{p}\left(1-\frac{\beta_{2}}{\beta_{1}}\|x\|^{\vartheta-p}\right) .
$$

Since $p<\vartheta$, we can find $\rho>0$ small enough such that

$$
\inf _{\|x\|=\rho}\{R(x)\}>0
$$

Because $\int_{Z} j(z, 0) d z \geqslant 0$, we have that $R(0) \leqslant 0$. Also for all $\xi>0$, we have

$$
R\left(\xi u_{1}\right)=\frac{\xi^{p}}{p}\left\|\nabla u_{1}\right\|_{p}^{p}-\frac{\lambda_{1} \xi^{p}}{p}\left\|u_{1}\right\|_{p}^{p}-\int_{Z} j\left(z, \xi u_{1}(z)\right) d z=-\int_{Z} j\left(z, \xi u_{1}(z)\right) d z,
$$

since $\left\|\nabla u_{1}\right\|_{p}^{p}=\lambda_{1}\left\|u_{1}\right\|_{p}^{p}$ (Rayleigh quotient). From the proof of Lemma 2 we know that

$$
\frac{j\left(z, \xi u_{1}(z)\right)}{\xi u_{1}(z)} \longrightarrow v_{+}(z) \text { almost everywhere on } Z \text {, as } \xi \rightarrow+\infty \text {. }
$$

So, recalling that $u_{1}(z)>0$ almost everywhere on $Z$, we have

$$
j\left(z, \xi u_{1}(z)\right) \longrightarrow+\infty \text { almost everywhere on } Z \text {, as } \xi \rightarrow+\infty \text {. }
$$

So for $\xi>0$ large enough we shall have that $R\left(\xi u_{1}\right) \leqslant 0$. This permits the use of Theorem 1 which gives us $x \in W_{0}^{1, p}(Z)$ such that $R(x)>0 \geqslant R(0)$ (hence $x \neq 0$ ) and $0 \in \partial R(x)$. From this last inclusion we obtain

$$
0=A x-\lambda_{1}|x|^{p-2}-v
$$

with $v \in \partial \psi(x) \subseteq L^{q}(Z)$. Hence

$$
A x=\lambda_{1}|x|^{p-2} x+v
$$

and

$$
\langle A x, \varphi\rangle=\lambda_{1}\left(|x|^{p-2} x, \varphi\right)_{p q}+(v, \varphi)_{p q} \quad \forall \varphi \in \mathcal{C}_{0}^{\infty}(Z)
$$


Here by $(\cdot, \cdot)_{p q}$ we denote the duality brackets for the pair $\left(L^{p}(Z), L^{q}(Z)\right)$. So, for all $\varphi \in \mathcal{C}_{0}^{\infty}(Z)$, we have

$$
\int_{Z}\|\nabla x(z)\|^{p-2}(\nabla x(z), \nabla \varphi(z))_{\mathbf{R}^{N}} d z=\int_{Z}\left(\lambda_{1}|x(z)|^{p-2} x(z)+v(z)\right) \varphi(z) d z .
$$

From the definition of the distributional derivative we have

$$
\left\{\begin{array}{l}
-\operatorname{div}\left(\|\nabla x(z)\|^{p-2} \nabla x(z)\right)=\left.\lambda_{1} x(z)\right|^{p-2} x(z)+v(z) \quad \text { almost everywhere on } Z \\
\left.x\right|_{\Gamma}=0
\end{array}\right.
$$

so

$\left\{\begin{array}{l}-\operatorname{div}\left(\|\nabla x(z)\|^{p-2} \nabla x(z)\right)-\left.\lambda_{1} x(z)\right|^{p-2} x(z) \in \partial j(z, x(z)) \quad \text { almost everywhere on } Z \\ \left.x\right|_{\Gamma}=0\end{array}\right.$

(recall that $v \in \partial \psi(x)$ implies $v(z) \in \partial j(z, x(z))$ almost everywhere on $Z$ ). Therefore $x \in W_{0}^{1, p}(Z)$ is a nontrivial solution of $(H V I)$.

REMARK 5. In a companion paper we shall deal with the existence of multiple solutions for semilinear (that is, $p=2$ ) hemivariational inequalities at resonance. Our formulation here incorporates problems with discontinuities. In this case $j(z, \zeta)=\int_{0}^{\zeta} h(z, r) d r$ with $h: Z \times \mathbb{R} \longmapsto \mathbb{R}$ a Borel measurable function. In this case we know (see Chang [6]) that $\partial j(z, \zeta) \subseteq\left[h_{1}(z, \zeta), h_{2}(z, \zeta)\right]$ with $h_{1}(z, \zeta)=\liminf _{\bar{\zeta} \rightarrow \zeta} h(z, \bar{\zeta})$ and $h_{2}(z, \zeta)=\limsup _{\bar{\zeta} \rightarrow \zeta} h(z, \bar{\zeta})$. Such problems were considered by Chang [6] for semilinear equations $(p=2)$.

\section{REFERENCES}

[1] S. Ahmad, A. Lazer and J. Paul, 'Elementary critical point theory and perturbations of elliptic boundary value problems at resonance', Indiana Univ. Math. J. 25 (1976), 933-944.

[2] A. Ambrosetti and P.H. Rabinowitz, 'Dual variational methods in critical point theory and applications', J. Funct. Anal. 14 (1973), 349-381.

[3] V. Benci, P. Bartolo and D. Fortunato, 'Abstract critical point theorems and applications to nonlinear problems with strong resonance at infinity', Nonlin. Anal. 7 (1983), 961-1012.

[4] H. Brezis, Analyse fonctionnelle. Théorie et applications (Masson, Paris, 1983).

[5] F. Browder and P. Hess, 'Nonlinear mappings of monotone type', J. Funct. Anal. 11 (1972), 251-294.

[6] K.C. Chang, 'Variational methods for nondifferentiable functionals and their applications to partial differential equations', J. Math. Anal. Appl. 80 (1981), 102-129.

[7] F.H. Clarke, Optimization and nonsmooth analysis (Wiley, New York, 1983).

[8] L. Gasiński and N.S. Papageorgiou, 'Existence of solutions and of multiple solutions for eigenvalue problems of hemivariational inequalities', Adv. Math. Sci. Appl. (to appear). 
[9] D. Goeleven, D. Motreanu and P.D. Panagiotopoulos, 'Multiple solutions for a class of eigenvalue problems in hemivariational inequalities', Nonlin. Anal. 29 (1997), 9-26.

[10] E. Landesman and A. Lazer, 'Nonlinear perturbations of linear elliptic value problems at resonance', J. Math. Mech. 19 (1970), 609-623.

[11] P. Lindquist, 'On the equation $\operatorname{div}\left(\|\nabla x\|^{p-2} \nabla x\right)+\lambda|x|^{p-2} x=0$ ', Proc. Amer. Math. Soc. (1990), 157-164.

[12] Z. Naniewicz and P.D. Panagiotopoulos, Mathematical theory of hemivariational inequalities and applications (Marcel-Dekker, New York, 1995).

[13] P.D. Panagiotopoulos, Hemivariational inequalities and their applications (Birkhäuser, Boston, 1988).

[14] P.H. Rabinowitz, 'Some minimax theorems and their applications to nonlinear partial differential equations', in Nonlinear Analysis: $A$ Collection of Papers in Honor of $E$. Rothe, (L. Cesari, R. Kannan and H. Weinberger, Editors) (Academic Press, New York, 1978), pp. 161-177.

[15] P.H. Rabinowitz, Minimax methods in critical point theory with applications to differential equations, CMBS Regional Conf. Series in Math. 65 (Amer. Math. Soc., Providence, R.I., 1986).

[16] R. Showalter, Hilbert space methods for partial differential equations (Pitman, London, 1977).

[17] K. Thews, 'Nontrivial solutions of elliptic equations at resonance', Proc. Roy. Soc. Edindurgh Ser. A 85 (1980), 119-129.

[18] J. Ward, 'Applications of critical point theory to weakly nonlinear boundary value problems at resonance', Houston J. Math. 10 (1984), 291-305.

Jagellonian University

Institute of Computer Science

ul. Nawojki 11

30072 Cracow

Poland

e-mail: gasinski@softlab.ii.uj.edu.pl

\author{
National Technical University \\ Department of Mathematics \\ Zografou Campus \\ Athens 15780 \\ Greece \\ e-mail: npapg@math.ntua.gr
}

